UCRL-JC-129713

PREPRINT

\title{
Achromatically Filtered Diamond Photoconductive Detectors for High Power Soft X-ray Flux Measurements
}

\author{
R. E. Turner \\ O. L. Landen \\ P. Bell \\ R. Costa
}

D. Hargrove

This paper was prepared for submittal to the

12th Topical Conference on High-Temperature Plasma Diagnostics

Princeton, NJ

June 7-11, 1998

June 2, 1998

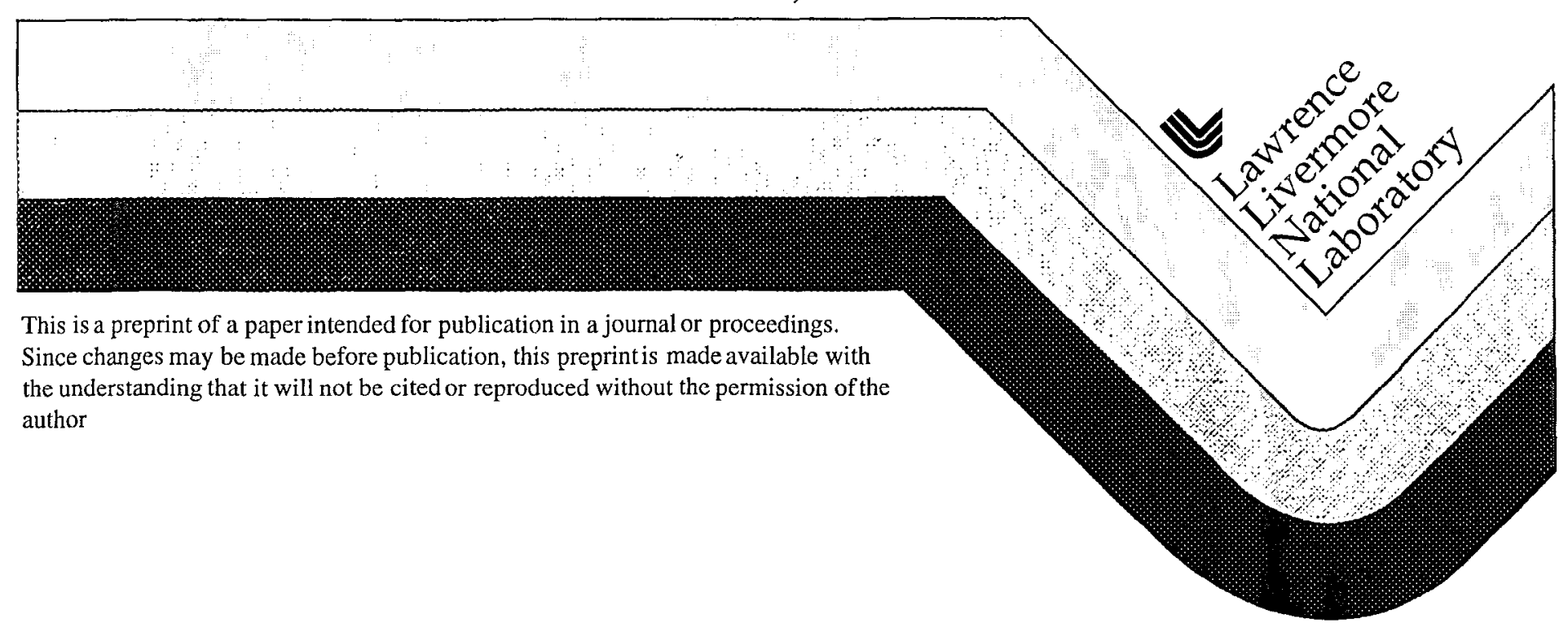




\section{DISCLAIMER}

This document was prepared as an account of work sponsored by an agency of the United States Government. Neither the United States Government nor the University of California nor any of their employees, makes any warranty, express or implied, or assumes any legal liability or responsibility for the accuracy, completeness, or usefulness of any information, apparatus, product, or process

disclosed, or represents that its use would not infringe privately owned rights. Reference herein to any specific commercial product, process, or service by trade name, trademark, manufacturer, or otherwise, does not necessarily constitute or imply its endorsement, recommendation, or favoring by the United States Government or the University of California. The views and opinions of authors expressed herein do not necessarily state or reflect those of the United States Government or the University of California, and shall not be used for advertising or product endorsement purposes. 


\title{
Achromatically filtered diamond photoconductive detectors for high power soft $x$-ray flux measurements.
}

R.E. TURNER, O.L. LANDEN, P. BELL, R. COSTA, D. HARGROVE Lawrence Livermore National Laboratory, Livermore, CA 94550

\begin{abstract}
A $1 \mathrm{~mm}$ square diamond photoconductive detector (PCD) has been installed on the LLNL Nova laser system, for use as a broad band soft x-ray power diagnostic. The PCD is installed behind an array of pinholes, which cast multiple, overlapping images of the source onto the diamond. This allows reduction of the x-ray intensity, to avoid saturation problems, while avoiding the spectral dependency of thin film filters. The diode current is read out on a $5 \mathrm{GHz}$ bandwidth scope. The system is calibrated by comparison to an absolutely calibrated array of filtered vacuum X-ray photodiodes (XRD's) ("dante"). The time response of the PCD and its bias electronics have been characterized using the 5 th harmonic $(210 \mathrm{~nm})$ of a short pulse $(<1$ ps) Ti:sapphire laser. The data show a fast rise, limited by the $5 \mathrm{GHz}$ scope bandwidth, and a slower fall off, characterized by an RC time of order 200 ps.
\end{abstract}

This work was performed under the auspices of the U.S. Department of Energy by Lawrence Livermore National Laboratory under contract No. W-7405-Eng-48. 


\title{
Achromatically filtered diamond photoconductive detectors for high power soft $x$-ray flux measurements.
}

\author{
R.E. TURNER, O.L. LANDEN, P. BELL, R. COSTA, D. HARGROVE \\ Lawrence Livermore National Laboratory, Livermore, CA 94550
}

\begin{abstract}
A $1 \mathrm{~mm}$ square diamond photoconductive detector (PCD) has been installed on the LLNL Nova laser system, for use as a broad band soft $x$-ray power diagnostic. The PCD is installed behind an array of pinholes, which cast multiple, overlapping images of the source onto the diamond. This allows reduction of the x-ray intensity, to avoid saturation problems, while avoiding the spectral dependency of thin film filters. The diode current is read out on a $5 \mathrm{GHz}$ bandwidth scope. The system is calibrated by comparison to an absolutely calibrated array of filtered vacuum x-ray photodiodes (XRD's) ("dante"). The time response of the PCD and its bias electronics have been characterized using the 5 th harmonic $(210 \mathrm{~nm})$ of a short pulse $(<1 \mathrm{ps})$ Ti:sapphire laser. The data show a fast rise, limited by the $5 \mathrm{GHz}$ scope bandwidth, and a slower fall off, characterized by an RC time of order 200 ps.
\end{abstract}


Photoconductive detectors (PCD) made from diamond have been shown $[1,2]$ to be a sensitive detector of soft $x$-rays. In addition, they possess the desirable characteristic of having a spectrally flat response [ 3 ]. However, for some applications, such as ICF, they are too sensitive; one must either use filters, which destroy the spectral flatness; move far away, which is frequently impractical; or attempt to apply a correction for the saturation [2] to the analysis. We report here the results of using an array of pinholes to reduce the flux to a small enough level that the device operates in the linear regime.

The geometry is shown schematically in figure 1 . A 16 by 16 square array of $10 \mu \mathrm{m}$ diameter pinholes, spaced $200 \mu \mathrm{m}$ apart, is placed 240 $\mathrm{cm}$ from the target. The $1 \mathrm{~mm}$ square PCD is placed $60 \mathrm{~cm}$ beyond the array, leading to a $4 x$ demagnification. The demagnification of $4 x$ was chosen becuase the $x$-ray sources we are usually interested in are spatially defined by a mm-scale laser entrance hole (LEH) of a hohlraum. These sources emit radiation with a characteristic black body temperature in the range of 100 to $250 \mathrm{eV}$. The pinhole array images this source onto the PCD, resulting in a minimum of 16 images. It is desirable that these images be overlapping so the PCD may be more uniformly illuminated. The combination of demagnification, pinhole separation and size, and stand-off distance between pinhole array and PCD has been chosen to allow both geometric and, in the case of photon energies below $1 \mathrm{keV}$, diffractive blurring to be effective in creating such overlap for mm-sized sources.

The flux is also reduced by a factor of 500, compared to the case where the pinhole array is removed. The factor of 500 was chosen to keep the PCD carrier density created by a $200 \mathrm{eV}, 1 \mathrm{~mm}$ diameter blackbody source at $3 \mathrm{~m}$ below $1 \mathrm{e} 16 \mathrm{cc}$, thus avoiding non-linear signal corrections due to carrier-carrier scattering [1]. This level of filtration also keeps the output signal at the volt level, much less than the bias voltage and hence obviating the need for impedance corrections [1]. We note that reducing the signal 500x without filter or pinhole array would have required an unrealistic PCD to source distance of $70 \mathrm{~m}$. Because only a small number of the 256 holes are 
used, the system is not sensitive to small misalignments - if the target or detector moves, a different row of holes is automatically used. Of course, there will be a change in the system response following small alignment changes if all of the array's holes are not the same size. Although the tolerance on one of the laser-drilled pinhole areas is $\pm 40 \%$, the error fluctates statistically, so for $\mathrm{n}$ different pinholes used by a new alignment, the contribution to pre-existing calibration uncertainty is $40 \% \times \sqrt{n} / 16$ (e.g. only $\pm 5 \%$ calibration uncertainty if displacement of view is one row $(n=4))$.

There are several other assumptions. (1) It is assumed that, within the LEH view, there are no small regions with much higher emissivity than the average. The images of such regions could locally saturate the PCD. Again, diffractive blurring is effective in mitigating this problem for the photon energies most likely to cause saturation (above the C K edge at 300-500 eV) (2) For LEH's considerably smaller than $1000 \mu \mathrm{m}$ in diameter, the system is somewhat sensitive to small misalignments, since images at photon energies not benefitting from diffractive blurring ( $>1 \mathrm{keV}$ ) no longer overlap. In this case, it is possible, with a small sideways misalignment, to lose part of a high energy image on one side, without gaining part of a new image on the opposite side. In the worst case of a point source, a small misalignment could cause the number of high energy images falling on the PCD to go from 16 to 9, if a side column and top row were simultaneously "lost" without being replaced on the opposite side. This problem can be fixed, if necessary, by changing to a higher magnification.

For a well aligned system, four holes from each of four rows of the array project onto the PCD, leaving six holes from each row on each side available for overlap coverage, for large images. If the image size becomes larger than six times the projected array hole spacing, then, even for well aligned systems, the average intensity on the PCD will begin to fall. In the geometric limit, this restricts us to source sizes $<6 \mathrm{~mm}$. In the diffraction limit, this requirement restricts us to photon energies $>60 \mathrm{eV}$. These limits can be extended by using larger arrays.

The high energy limit for this system is determined by either transmission through the PCD, or transmission through the pinhole 
array substrate. For our components $(2 \mathrm{~mm}$ thick PCD, 50 um thick Ta pinhole substrate) both effects begin to be non-negligible when the photon energy approaches $9 \mathrm{keV}$.

One further caution needs to be mentioned. If the flux just above the carbon $\mathrm{K}$ edge is appreciably larger than the flux characteristic of a several hundred $\mathrm{eV}$ black-body, it may locally saturate the diamond, since its absorption depth is small $(60 \mathrm{~nm})$, and thus yield a lower than expected signal.

In operation, the PCD has 800 volts applied through a bias resistor. The signal is capacitively coupled into an air-dielectric high bandwidth coaxial cable, and into a Tektronix SCD 5000 oscilloscope. In Nova operation, a typical signal is a few volts.

We have measured the time response of the PCD-bias ' $T$ ' electronicsscope combination, using a short pulse $(<1 \mathrm{ps}), \mathrm{uv}(210 \mathrm{~nm})$ laser (5th harmonic of Ti:sapphire laser). The cable has been separately characterized. These photons are just above the $5.5 \mathrm{eV}$ diamond bandgap, and thus can directly excite the detector. The results are shown in figure 2 , which shows a scope limited ( $5 \mathrm{GHz} \mathrm{BW}$ ) rise, followed by a slower $(1 / \mathrm{e}=200 \mathrm{ps})$ decay. We use this data to temporally deconvolve shot data, using the usual Fourier transform techniques.

Finally, we have calibrated the pinhole-PCD combination in situ, by firing a Nova laser beam at a gold disk. The disk's normal is centered between the line of sight to the PCD and the Dante (calibrated x-ray diodes) diagnostic [4], and we presume the flux to each instrument is identical. The Dante calibration has been discussed elsewhere [4]. The results are shown in figure 3. The slightly higher PCD signal late in time (after the laser is off) may be due to the Dante's lack of sensitivity to very soft $(<80 \mathrm{eV})$ photons. For the system described here, the pinhole array - PCD sensitivity is $198 \mathrm{GW} / \mathrm{ster} /$ volt (into $50 \mathrm{ohms}$ ).

We are currently using the detectors on the Nova and Omega systems. Among their advantages are broadband spectral response; multi-wavelength calibrations are not needed, nor are complicated spectral unfold procedures. Their compact physical size allows 
placement as needed. For example, PCD's mounted at varying angles can be used to sample the radiation flux experienced by an experimental package mounted to a hohlraum side-wall. They are also easily mounted to view into the laser entrance hole of a holraum. This has been shown to be a better procedure for obtaining the drive experienced by a central capsule than viewing the hohlraum mid-plane [5]. Figure 4 shows Omega data from both a Dante detector system, and a co-linearly mounted pinhole arrayPCD, viewing into the LEH. The PCD was calibrated against the Dante on a similar, but different, shot. We observe that both diagnostics show very good agreement with each other, as a function of time.

Still another application is to use pinhole arrays to reduce the signal on Dante-type systems, to allow them to be mounted at a physically closer, more convenient distance, for measuring high power fluxes. For weak signals, the dynamic range can be extended several orders of magnitude by simply removing the array. We envision such a system will find use on NIF, for measuring the high contrast shaped pulse necessary for ignition targets. 


\section{REFERENCES}

1. L.S. Pan, D.R. Kania, P. Pianetta, and O.L. Landen, Appl. Phys. Lett. 57, 623, (1990).

2. D.R. Kania, L. Pan, H. Kornblum, P. Bell, O.N. Landen, and P. Pianetta, Rev.Sci.Instrum. 61, 2765 (1990).

3. D.R. Kania, L.S. Pan, P. Bell, O.L. Landen, H. Kornblum, P. Pianetta, and M.D. Perry, J. Appl. Phys. 68, 124 (1990).

4. H.N. Kornblum, R.L. Kauffman, and J.A. Smith, Rev. Sci. Instrum. 57,2179 (1986).

5. C. Decker, R.E. Turner, O.L. Landen, L.J. Suter, P. Amendt, H.N. Kornblum, B.A. Hammel, T.J. Murphy, J. Wallace, N.D. Delamater, P. Gobby, A.A. Hauer, G.R. Magelssen, J.A. Oertel, J. Knauer, F.J. Marshall, D. Bradley, W. Seka, and J.M. Soures, Phys. Rev. Lett. 79, 1491 (1997). 


\section{FIGURE CAPTIONS}

Figure 1. Geometry of the x-ray source, pinhole array, and detector (not to scale).

Figure 2. Impulse response of the PCD, bias electronics, and $5 \mathrm{Ghz}$ scope, using a short pulse uv laser as the excitation source.

Figure 3. The soft x-ray emission from a Nova gold disk shot, used to calibrate the PCD to the dante diagnostic.

Figure 4. Co-located pinhole array-PCD and Dante measure $\mathrm{x}$-ray flux from the laser entrance hole of a hohlraum on Omega. 


\section{Geometry of source-pinhole array-PCD (not to scale)}

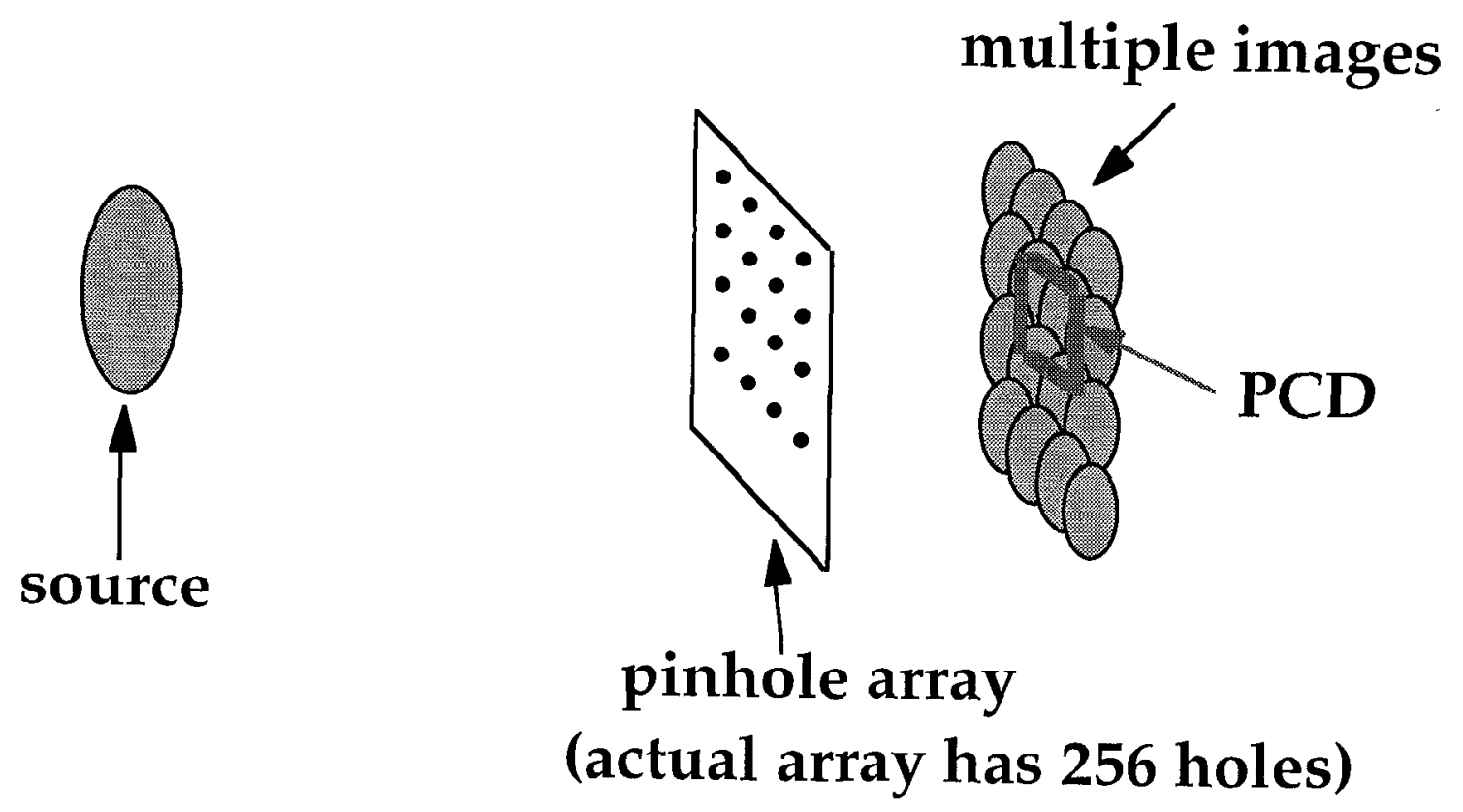

Figure 1 


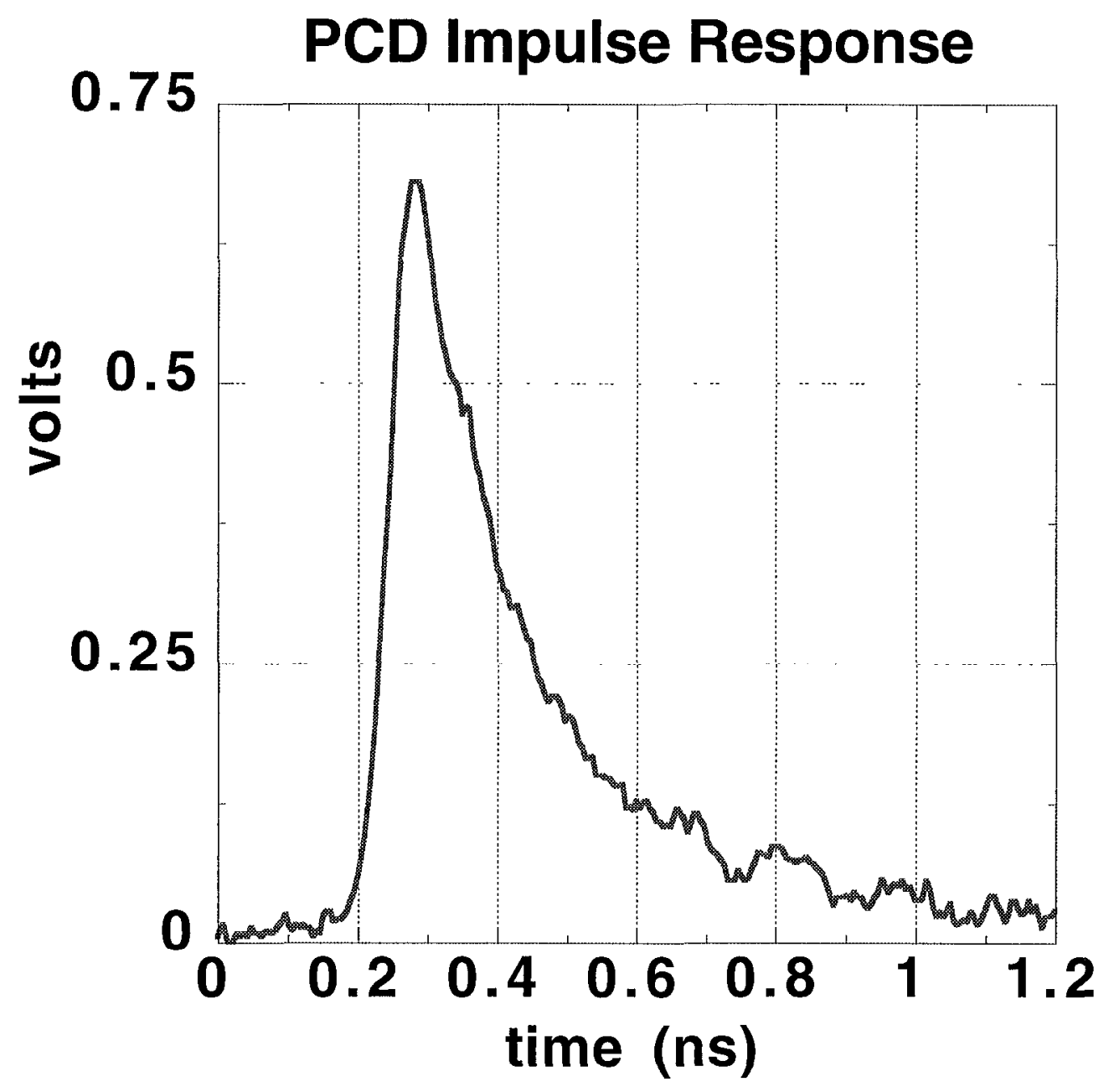

Figure 2 


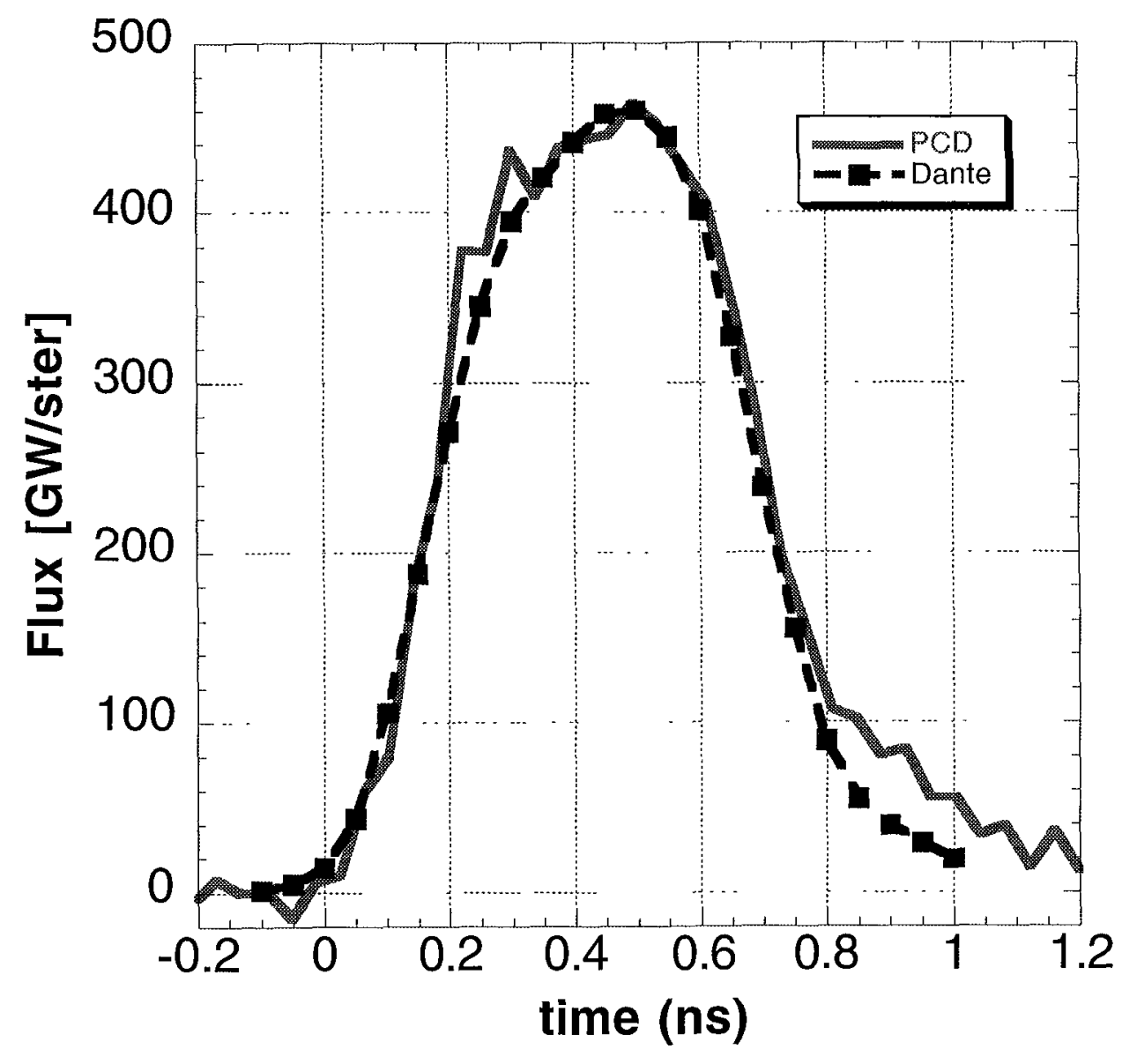

Figure 3 


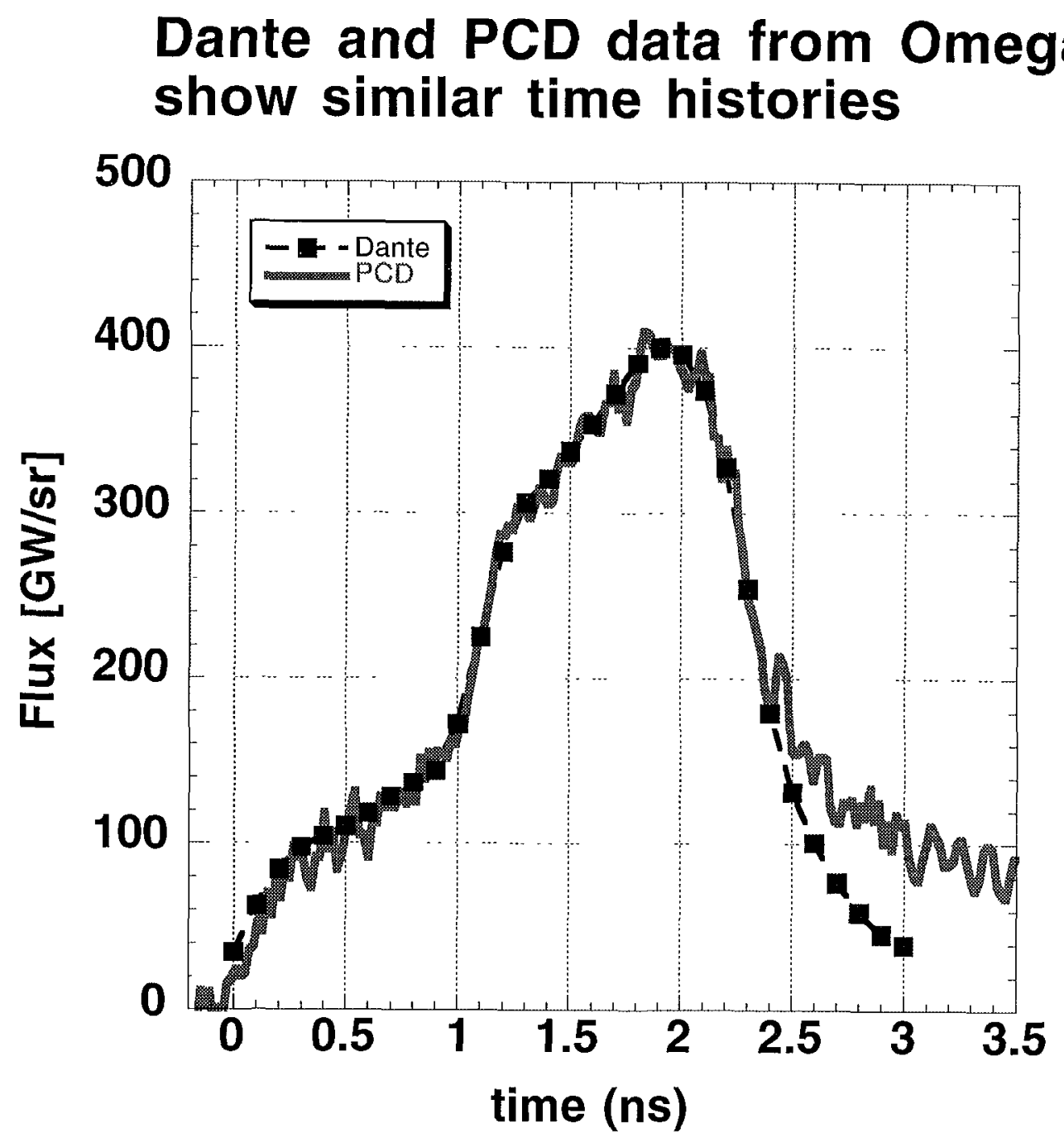

Figure 4 


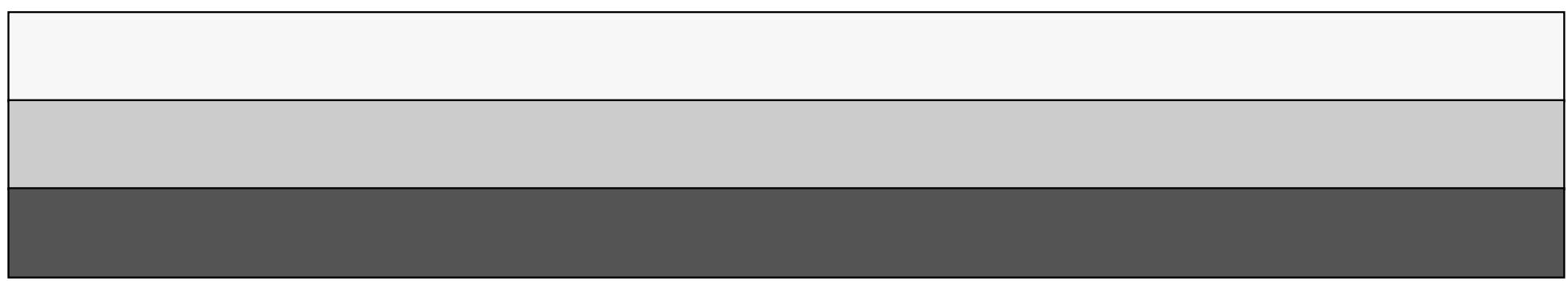

